(C) [2006] IEEE. Reprinted, with permission, from [Peter Leijdekkers and Valerie Gay,Personal Heart Monitoring and Rehabilitation System using Smart Phones, Mobile Business, 2006. ICMB '06. International Conference on, 26-27 June 2006]. This material is posted here with permission of the IEEE. Such permission of the IEEE does not in any way imply IEEE endorsement of any of the University of Technology, Sydney's products or services. Internal or personal use of this material is permitted. However, permission to reprint/republish this material for advertising or promotional purposes or for creating new collective works for resale or redistribution must be obtained from the IEEE by writing to pubs-permissions@ieee.org. By choosing to view this document, you agree to all provisions of the copyright laws protecting it 


\title{
Personal Heart Monitoring and Rehabilitation System using Smart Phones
}

\author{
Peter Leijdekkers, Valérie Gay \\ Faculty of IT, University of Technology Sydney \\ UTS FIT, PO box 123, Broadway 2007 NSW Australia \\ E-mail: [peterl,valerie]@it.uts.edu.au
}

\begin{abstract}
This paper discusses a personalized heart monitoring system using smart phones and wireless (bio) sensors. Based on several scenarios we present the functionality of a prototype we have built. The application is capable of monitoring the health of high risk cardiac patients. The smart phone application analyses in real-time sensor and environmental data and can automatically alert the ambulance and pre assigned caregivers when a heart patient is in danger. It also transmits sensor data to a healthcare centre for remote monitoring by a nurse or cardiologist. The system can be personalized and rehabilitation programs can monitor the progress of a patient. Rehabilitation programs can be used to give advice (e.g. exercise more) or to reassure the patient.
\end{abstract}

\section{Introduction}

Cardiovascular disease is the leading cause of mortality in the developed world. Cardiovascular disease refers to various medical conditions that affect the heart and the blood vessels. These conditions include coronary artery disease, heart attack, myocardial infarction (MI), angina, congestive heart failure, hardening of the arteries, stroke and peripheral vascular disease. The risk factors for developing cardiovascular diseases can be medical but also lifestyle related [1]. Major risk factors are:

- High Blood Pressure

- Diabetes Mellitus

- High Blood Cholesterol and other Lipids

Being overweight or obese also contributes to developing cardiovascular diseases. Obesity is becoming a major problem in developed countries and the World Health Organisation (WHO) estimates that 1 billion adults are overweight and at least 300 million of them are obese [2]. Finally smoking (tobacco) is another lifestyle factor contributing to developing cardiovascular diseases.

With an ageing population, cardiovascular diseases will only increase over the next 10 to 15 years since it predominantly occurs in the age group of 55 and over [1]. The estimated direct and indirect cost of cardiovascular diseases in the United States alone is $\$ 393.5$ billion for 2005 according to the American Heart Association [1].

To reduce the cost and the anxiety of people with known cardiovascular diseases we propose a portable monitoring system that monitors the heart and notifies the person or external party in case of abnormalities. Our monitoring system is meant for patients that are at risk and need to be monitored continuously. Our system focuses on the following key aspects:

- Monitoring: Continuously heart monitoring using an ECG sensor. In case of a heart failure the monitoring device should immediately call an ambulance and warn caregivers. Also the location of the person should be reported automatically so that ambulance services can be directed to the patient.

- Cardiac rehabilitation: This aims at maximizing physical, psychological and social functioning to enable patients to live productively and with confidence [4]. Our system aims at monitoring and encouraging the patient to perform his/her physical exercises as well as improving confidence by constant monitoring. Underlying causes for the cardiovascular diseases are often high blood pressure, being overweight or a high cholesterol level. Cardiac Rehabilitation aims at making lifestyle changes such as getting more active, loosing weight, reducing stress or quit smoking.

Our objective is to investigate and develop a portable application whereby a heart patient is monitored using one or more (wireless) sensors. The 
sensor data is collected and transferred wirelessly to a smart phone.

A state-of-the-art on heart monitoring solutions can be found in [6]. Our solution is similar to AMON [7], MOLEC[8] and Epi-medics [9] which analyse the ECG signal on the local device. One distinction of our solution compared to the others is that we can personalize the monitoring and we have mechanisms in place to locate the user in case of emergency. We detect life threatening arrhythmias and give the patient general information about their health when they are not in a dangerous situation. We also include rehabilitation applications that can be personalized for the patients and monitor their progress.

This paper presents an overview of our monitoring system to date. We focus in this paper on the usage of the application by a heart patient. Section 2 describes various scenarios each describing a typical situation and illustrates how our prototype is used. Section 3 gives the status of our prototype and research work. Section 4 concludes this paper.

\section{Scenarios}

This section discusses several scenarios showing the daily use of our application from the perspective of a patient and a health centre. John Smith has had a heart attack and is about to leave the hospital. John is very concerned that he will be struck by another heart attack and his concerns are confirmed by the American Heart Association indicating that people who've had a heart attack have a sudden death rate that is 4 to 6 times that of the general population [1]. To reduce his anxiety the cardiologist offers John the possibility to borrow a smart phone and sensors that will monitor him 24 hours a day.

\subsection{Seeing the cardiologist}

The cardiologist performs several checks on John to determine which sensors he needs and the threshold levels for each sensor.

An ECG recording is made and the average heart rate is determined for John. Because of John's age and his recent heart attack the cardiologist decides that the heart rate should not go over 120 bpm for the next 30 days. Furthermore, the ECG diagram shows that John suffers from Premature Ventricular Contractions (PVC) which in itself is not a problem but needs to be monitored.
The blood pressure is taken and John suffers from moderate hypertension. His blood pressure needs to come down and the cardiologist prescribes a blood pressure-reducing medication. The cardiologist determines that John should monitor his blood pressure three times a day.

John's weight is measured and the cardiologist determines his body mass index (BMI). It turns out that John is overweight and needs to loose weight in the abdominal area. The cardiologist puts him on a DASHdiet which promotes fruits, vegetables, whole grains and low-fat dairy while limiting saturated fat and red meat. He also recommends John to start exercising moderately every day as advised by the Australian Heart Foundation [5].

The cardiologist measures his cholesterol level (which is okay) and confirms with John that he is not diabetic.

The cardiologist is now ready to configure the heart monitor application and cardiac rehabilitation application directly on the smart phone. He decides that John will have to wear an ECG sensor day and night and needs to measure his blood pressure and weight several times a day. The applications are password protected so that only a cardiologist can configure the sensors and sensor specific settings.

Figure 1 illustrates how John's details are entered into the application on the smart phone. The Weight Monitor and Blood Pressure Monitor applications are activated.

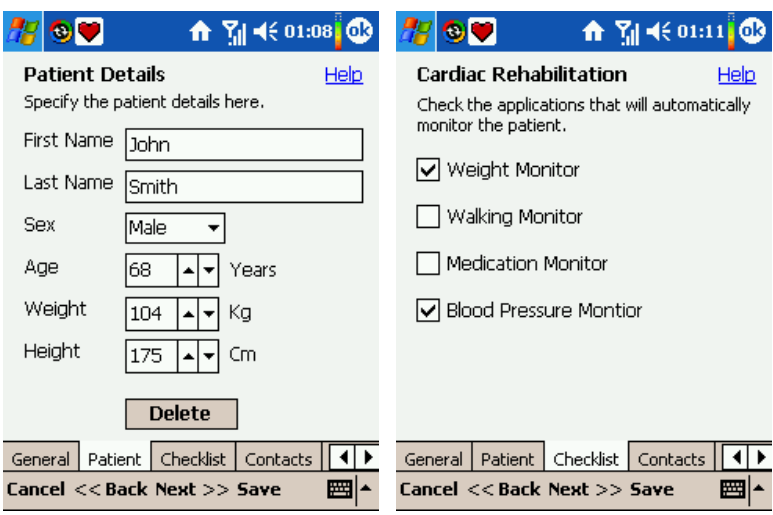

Figure 1: Patient specific configuration

Since John has bad eye sight, but luckily no hearing problem, the cardiologist tailors the user interface to interact with John using sound and vibrations (John will be able to change those settings when desired). 


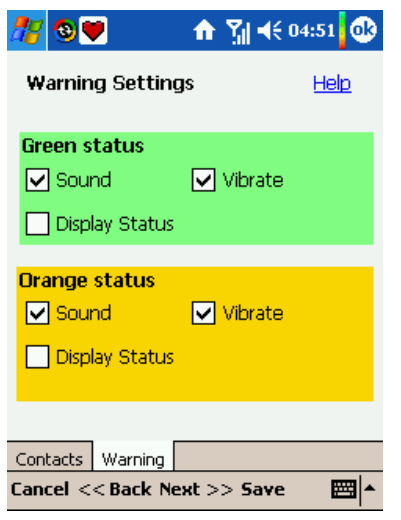

Figure 2: Tailoring the user interaction mode

Since the sensors use Bluetooth communication to transfer the data wirelessly to the smart phone, the cardiologist needs to configure the sensors he will borrow to John. Figure 3 (left) illustrates this process. The cardiologist selects the desired sensor from the dropdown list and turns on the corresponding sensor. The smart phone will automatically scan for Bluetooth devices when the search button is clicked. The cardiologist then assigns the sensor to the specific Bluetooth sensor found and sets the corresponding COM-port and Baud-rate. He can also specify the polling rate indicating how fast data should be collected from the sensor. The application has build-in security features that will automatically reconnect a sensor in case of connection loss.

In Figure 3 (right) we can see that the cardiologist sets the warning level to $120 \mathrm{bpm}$ to automatically warn the patient and the cardiologist. He sets the threshold level to $150 \mathrm{bpm}$ for calling the emergency services. Under no circumstances should John's heart rate go over $150 \mathrm{bpm}$ for the next 30 days.

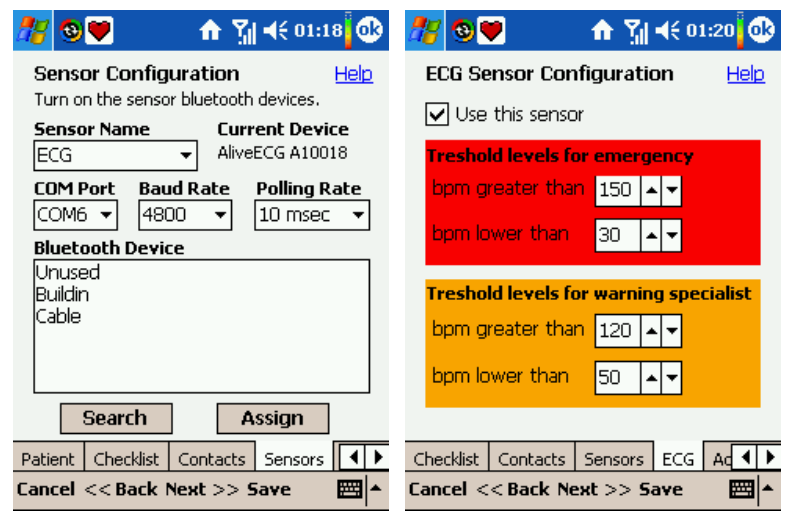

Figure 3: Sensor configuration

The cardiologist configures the blood pressure monitor and weight monitor in a similar way. In addition, he configures when John should measure his blood pressure (morning, after lunch and evening). The application will automatically remind John when its time to measure his weight or blood pressure.

The cardiologist then explains to John how to attach the ECG monitor to his body and in particular how to place the electrodes correctly.

He also demonstrates how to take the blood pressure correctly. Since the blood pressure monitor and scale are Bluetooth enabled, John only needs to push one button on each device to have the values automatically transmitted to his smart phone.
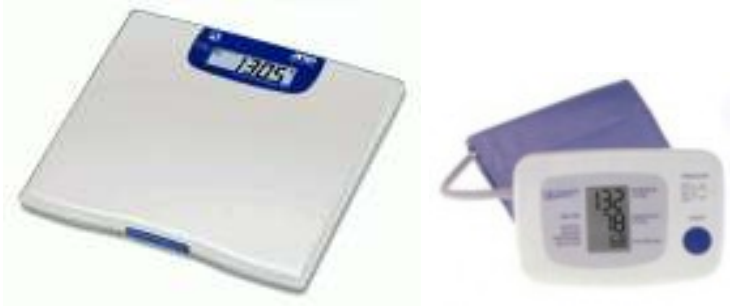

Figure 4: A\&D Scale and Blood Pressure Monitor

\subsection{A normal day for the patient}

John is back home for a week and happy to be in his own environment. He also appreciates that other people do not see that he is wearing an ECG sensor (Figure 5, right). He uses the smart phone the cardiologist borrowed him as his everyday mobile phone.

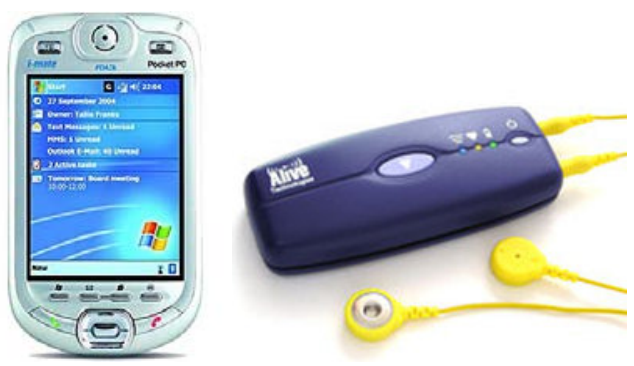

Figure 5: i-mate Smartphone and Alive ECG sensor

After a good night's sleep, John wakes up and checks if the ECG electrodes are still properly attached to his body. The smart phone stayed in the cradle/charger overnight and the battery is fully charged. John feels more secure since he is monitored around the clock and he knows that if something goes wrong, the ambulance and the cardiologist will be contacted immediately.

As directed by the cardiologist, John checks his weight and blood pressure and keeps a log of what could explain a change in the readings (e.g. positive stress: birth of a grand child). Keeping a $\log$ is achieved by a simple interface on the smart phone 
where John can select an activity from a dropdown menu. The benefit of a log is that it allows the cardiologist to understand what activities John has done which could have caused a change in the blood pressure reading or weight.

John checks the blood pressure and weight according to the schedule prescribed by the cardiologist. The heart monitor application will remind him when a new check is due.

The cardiologist advised John to walk everyday for 30 minutes. The cardiologist configured the smart phone to be connected to a Bluetooth GPS receiver and John activates the GPS receiver when he is ready. The GPS and the application can track John while he is walking in the park and in clear sight of GPS satellites.

After the walk, John decides to say hello to his mates at the bowling club and have lunch with them. Since it is John's first visit to the bowling club since his operation, he records and senses this new location on his smart phone. The cardiologist told him to do so for all indoor locations he frequently visits.

WiFi and GSM can be used to determine the location when indoors [12]. Since GSM cellid and $\mathrm{WiFi}$ access points are not automatically related to a location, John has to 'relate' the Bowling club with the WiFi/GSM cellid data. Figure 6 shows how John can automatically spot WiFi access points and GSM cellids and assign these to the address of his bowling club. This information will allow the ambulance to locate him in case John has a second heart attack.

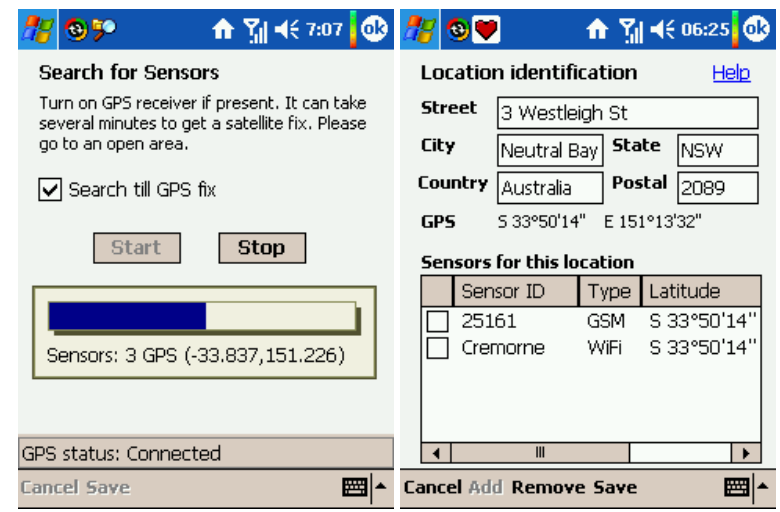

Figure 6: Location configuration

Back home, John decides to watch a movie. During that time, he places the smart phone in its cradle to charge the battery (the smart phone battery lasts for approximately 8 hours). As long as he stays within 10 meters of the smart phone he is monitored.

When John places the smart phone in the cradle, which is connected via his PC to the internet, the application automatically uploads the recorded data to the health centre database. The application records normal and non urgent warnings and can download new configuration data to the smart phone application as set by the cardiologist.

Half way through the movie, someone knocks on his door and John goes towards the door. However, he moves out of the smart phone's Bluetooth range and the smart phone plays loudly a message stating that the heart monitor is disconnected. John quickly grabs the smart phone and welcomes his visitor.

Towards the evening, the smart phone indicates that the ECG sensor battery is low. Since he is relaxed and feels well, he decides to change the ECG battery immediately. This takes only 1 minute and the battery lasts for approx 60 hours and he recharges the empty battery.

Some sensor threshold levels are set to congratulate or encourage John. For example, when John lost his first kilo, two days ago, the Weight monitor application congratulated him. Sensor measurements that are dangerously out of range will be transmitted directly to the health centre via GPRS and not wait till the next synchronization. This allows for the cardiologist or nurse to react immediately. When a blood pressure reading is out of range compared to a previous reading, the application will instruct John to measure the blood pressure again to avoid incorrect readings.

\subsection{Healthcare centre}

The healthcare centre has an application installed which is able to deal with users of the heart monitor application. John has been registered with this healthcare centre application and if one of the indicators is out of range, as set by the cardiologist, the application will alert the nurse in charge. The nurse will check his health record and in this case checks his history of blood pressure readings.

If the nurse identifies a dangerous situation, a cardiologist will be contacted immediately. In John's case, it was a minor warning and she just contacted John to take another blood pressure measurement. It turned out that he had put the cuff wrongly and the new reading was within the normal range.

John visits the healthcare centre after two weeks and discusses any concerns he has with his health. It is also the opportunity to change some of the parameters of the heart monitor. John is irritated by the fact that the application keeps reminding him to exercise more. He knows that he doesn't walk enough but he is just not confident enough and afraid that his heart rate will go too high. The cardiologist adjusts the settings and sets him a lower objective with the promise that John will do his best. 
The cardiologist also checks the blood pressure using the centres' blood pressure monitor. Since John's blood pressure readings vary significantly the cardiologist explains again to John how to properly place the cuff to obtain a correct reading.

\subsection{Emergency}

One day, John is walking his dog in the park when he starts feeling a strong pain in the chest. At the same time the smart phone detects a ventricular fibrillation ${ }^{1}$ rhythm which is a life threatening arrhythmia. John collapses and passes out. The smart phone plays loudly a message notifying John (Figure 7, left). If John was feeling well he would have had the opportunity to disable the alarm but this is not the case.

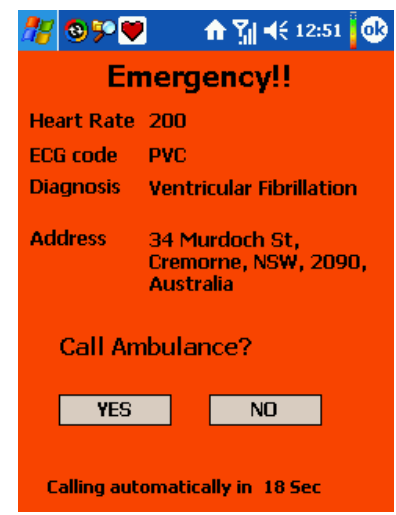

Figure 7: Emergency form

The smart phone automatically dials 000 and plays a recorded message stating John's name, the emergency and location. Also an SMS is send to the cardiologist and caregivers to warn them. Since GPS is activated the ambulance gets the longitude/latitude coordinates. While the ambulance is on its way, a message is played continuously on the smart phone. It says that John is having a heart attack and plays a first aid message instructing (potential) bystanders what to do. The ambulance reaches John in time and was able to treat him successfully by applying a defibrillator to the chest

\section{Prototype}

The scenarios described above are based on the prototype we are developing at the University of Technology, Sydney.

\footnotetext{
${ }^{1}$ A lethal arrhythmia characterized by the rapid, chaotic movements of the heart muscle that causes the heart to stop functioning and leads quickly to cardiac arrest.
}

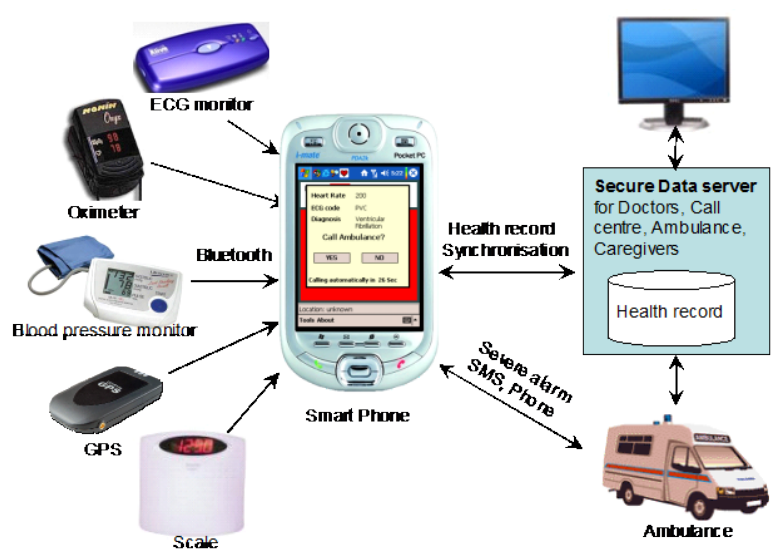

Figure 8: Architecture

We developed the personalized heart monitoring application on Microsoft's Windows Mobile Pocket PC platform. We selected this platform due to easy access to lower level APIs which are needed for the sensor manager modules. Also the tight integration with the operating system allows easier access to other applications running on the mobile device such as the calendar application, WiFi and obtaining the GSM cellid. We used the .Net Compact Framework extended with OpenNETCF [13] modules to build the application. The data is stored in an SQL CE Server which is a compact database for mobile devices.

We use a Bluetooth ECG/Accelerometer sensor from Alive Technologies [11] to detect life threatening arrhythmias and to detect falls. We focused on the ECG sensor and developed a heart beat detector and classifier complemented with a Ventricular Fibrillation and Ventricular Tachycardia detection algorithm [14]. The algorithms have been tested on ECG files from the MIT/BIH Arrhythmia Database [15]. This is a commonly used database to test and study different types of arrhythmias. The heart beat detector and classifier has a sensitivity value of $99.42 \%$ and a positive predictive value of $99.51 \%$ when tested against the MIT/BIH Arrhythmia records. This is a high level of accuracy and the algorithm is also capable of processing the live ECG data in real-time.

We use a Bluetooth enabled Blood Pressure Monitor and Scale from A\&D Medical [10] to be used with the Blood Pressure Monitor and Weight Monitor application which are currently prototyped.

The healthcare centre application is based on Microsoft .Net technology using IIS and SQL Database. We use ASP.net to create dynamic web applications.

We are currently focusing on the personalization aspect in cooperation with cardiac specialists. We are refining which information should be displayed 
depending on the type of patient. We are also developing an algorithm that uses the accelerometer data to accurately determine whether a patient has fallen or not.

In the near future we plan to conduct trials on cardiac patients in cooperation with a Sydney hospital to determine the feasibility and reliability of our prototype. This will provide us with valuable feedback to improve the application.

\section{Conclusion}

This paper described a 24/7 personalized heart monitoring using smart phones and wireless wearable sensors. Important features of the application are the following:

- the analysis of the sensor data on the local device,

- the possibility to call for external help and locate the patient in case of an emergency,

- $\quad$ high level of personalization.

- $\quad$ keeps a record of the data for further analysis by the healthcare centre.

By processing the data locally on the smart phone, a patient is monitored and reassured without needing to be continuously connected to a healthcare centre. This reduces the workload of medical staff, communication costs and motivates the patient's self-care.

The connection to a healthcare centre is important since there are limits to what can be processed locally and monitored automatically. We need the expertise of medical staff to complement our application.

The application provides valuable 24/7 sensor data which the medical staff can use to identify a problem and give a diagnosis. They can crosscheck, validate, and fine tune the personalization of the smart heart application to tailor it to a particular patient.

An issue will be the acceptance of our application by a health centre and medical specialists if we want the application to be used on a large scale.

We believe that our system is a low intrusive 24/7 heart monitoring application which will promote patient's autonomy and by providing personalized advice we hope that it will give the patients more confidence and improve their quality of life.

\section{Acknowledgements}

The authors would like to thank Ronny Haryanto for his contribution to the prototype.

\section{References}

[1] American Heart Association. Heart Disease and Stroke Statistics - 2005 Update. Dallas, Texas: American Heart Association; 2005.

[2] World Health Organization (who), Obesity and Overweight, http://www.who.int/dietphysicalactivity/publications/fa cts/obesity/en/, [last accessed 16th February 2006].

[3] Australian Institute of Health and Welfare (AIHW) 2004. Heart, stroke and vascular diseases - Australian facts 2004. AIHW Cat. No. CVD 27. Canberra: AIHW and National Heart Foundation of Australia (Cardiovascular Disease Series No. 22). http://www.aihw.gov.au/publications/cvd/hsvd04/hsvd 04.pdf.

[4] Australian Institute of Health and Welfare 2003. Secondary prevention and rehabilitation after coronary events or stroke: a review of monitoring issues. AIHW Cat. No. CVD 25. Canberra: Australian Institute of Health and Welfare.

http://www.aihw.gov.au/publications/cvd/sprces/sprces .pdf [last accessed 1st February 2006].

[5] Physical Activity Recommendations for People with Cardiovascular Disease http://www.heartfoundation.com.au/index.cfm?page $=4$ 2 [last accessed 1st February 2006].

[6] Peter Leijdekkers, Valérie Gay, 'Personal Heart Monitoring System using Smart Phones to detect life threatening Arrhythmias', 19th IEEE/ACM International Symposium on Computer-Based Medical Systems, CBMS 2006, Salt Lake City, Utah. Jun 22-23, 2006 .http://cbms2006.ece.byu.edu/index.html.

[7] Anliker, U., Ward, J.A., Lukowicz, P., Troster, G., Dolveck, F., Baer, M., Keita, F.; Schenker, E.B., Catarsi, F., Coluccini, L., Belardinelli, A., Shklarski, D., Alon, M., Hirt, E., Schmid, R.and Vuskovic, M., AMON: a wearable multiparameter medical monitoring and alert system, IEEE Transactions on Information Technology in Biomedicine, Volume 8, Issue 4, Dec. 2004 Page(s): 415 - 427.

[8] Rodriguez, J., Goni, A and Illarramendi, A., Real-time classification of ECGs on a PDA, Information Technology in Biomedicine, IEEE Transactions on, Volume 9, Issue 1, March 2005 Page(s): 23 - 34 .

[9] Rubel P. et Al, Towards personal eHealth in cardiology. Results from the EPI-MEDICS telemedicine project, Journal of Electrocardiology 38 (2005) pp.100-106

[10] A\&D Medical website http://www.andmedical.com.au/ [last accessed 2nd April 2006]

[11] Alive Technologies, http://www.alivetec.com/ [last accessed 1st February 2006]. 
[12] Leijdekkers, P. and Gay, V., Personalized Service and Network Adaptation for Smart Devices, IEEE APCC Asia Pacific Conference on Communications 2005. Perth, Australia, October 2005.

[13] OpenNETCF.org, 'The Premier .NET Compact Framework Shared Source Site' http://www.opennetcf.org, assessed March 2005.
[14] Ayesta U., Serrano L., Romero I., Complexity Measure revisited: A new algorithm for classifying cardiac arrhythmias, 23rd Annual International Conference of the IEEE Engineering in Medicine and Biology Society (2001).

[15] PhysioBank, ANSI/AAMI EC13 Test Waveforms, http://physionet.org/physiobank/database/aami-ec13/, [last accessed 1st February 2006]. 